\title{
Isotropization in the approach to big rip singularities for Cardassian models
}

\author{
Nikolaus Berndt ${ }^{1}$ and Alan D Rendall ${ }^{2}$ \\ ${ }^{1}$ Institut für Physik, Humboldt-Universität zu Berlin, Newtonstrasse 15, 12489 Berlin, Germany \\ 2 Max-Planck-Institut für Gravitationsphysik, Albert-Einstein-Institut, Am Mühlenberg 1, \\ 14476 Potsdam, Germany
}

Received 24 April 2008

Published 24 June 2008

Online at stacks.iop.org/CQG/25/145007

\begin{abstract}
Cardassian models are an alternative to general relativity which have been proposed as an approach to explaining accelerated cosmic expansion while avoiding directly introducing dark energy. They are generally formulated only in the homogeneous and isotropic case. In this paper an extension of the usual formulation to general spatially homogeneous geometries is given. A characteristic feature of many classes of Cardassian models is the occurrence of big rip singularities where the scale factor tends to infinity after a finite time. It is shown that big rip singularities are also widespread in more general homogeneous cases. It is also shown that there is isotropization in the approach to a big rip singularity which bears a strong resemblance to the late-time isotropization observed in cosmological spacetimes which accelerate forever in the future.
\end{abstract}

PACS number: 98.80.Jk

\section{Introduction}

The realization, based on observational data, that the expansion of the universe is accelerating has led to a lot of work which modifies traditional cosmological models by introducing exotic types of matter or replacing general relativity by something else. The focus of interest in the following is an example of the second approach. It concerns the Cardassian models which were first introduced in [3]. The standard solutions of the Einstein equations applied in cosmology are those which are homogeneous and isotropic. These are the FriedmannLemaitre-Robertson-Walker (FLRW) models. Often attention is concentrated on the spatially flat case, where the spacetime metric takes the form

$$
-\mathrm{d} t^{2}+a^{2}(t)\left(\mathrm{d} x^{2}+\mathrm{d} y^{2}+\mathrm{d} z^{2}\right)
$$


with scale factor $a(t)$. In these solutions a central role is played by the Friedmann equation

$$
\left(\frac{\dot{a}}{a}\right)^{2}=\frac{8 \pi \rho}{3}+\frac{\Lambda}{3},
$$

where $\rho$ is the energy density, $\Lambda$ is the cosmological constant and geometrized units are used where the gravitational constant and the speed of light take the numerical value unity. The other independent Einstein equation is the evolution equation

$$
\frac{\ddot{a}}{a}=-\frac{4 \pi}{3}(\rho+3 p)+\frac{\Lambda}{3}
$$

where $p$ is the pressure. As a consequence of energy-momentum conservation the matter quantities $\rho$ and $p$ satisfy

$$
\dot{\rho}+3 H(\rho+p)=0,
$$

where $H=\dot{a} / a$ is the Hubble parameter. It is well known that if equation (2) is satisfied at some time $t_{0}$ and equations (3) and (4) are satisfied at all times then (2) is satisfied at all times. This is a special case of the general phenomenon of propagation of the constraints in solutions of the Einstein equations. Accelerated expansion means that $\ddot{a}>0$ and it follows from (3) that this is only possible if $\Lambda>0$ or $\rho+3 p<0$. Many matter models in general relativity satisfy the strong energy condition $\rho+3 p \geqslant 0$ which corresponds to the fact that gravity is attractive. Accelerated expansion is only possible in general relativity if either $\Lambda>0$ or there is a matter field (dark energy) which violates the strong energy condition. The idea of [3] is to replace general relativity by another theory so as to make accelerated cosmological expansion possible with a vanishing cosmological constant and without the need for matter violating the strong energy condition.

In the Cardassian models of [3], which are formulated for homogeneous and isotropic geometries, the Friedmann equation (2) is replaced by the equation

$$
\left(\frac{\dot{a}}{a}\right)^{2}=\frac{8 \pi}{3}(\rho+f(\rho))
$$

for a smooth function $f$. The standard choice for $f(\rho)$ has the power-law form $B \rho^{n}$ for constants $B$ and $n$ but in principle the formalism can be developed for any function $f$. In later work [4] this approach was changed and the Cardassian term is only a function of part of the energy density of the matter, the rest energy density. This splitting requires the input of more information than the energy-momentum tensor alone. The analysis in this paper will concentrate on the original version although the later version will be commented on.

In [3] a possible origin of the additional term in the Cardassian framework was discussed in the context of braneworld models. A Cardassian term also arises in loop quantum cosmology as a manifestation of the discreteness of spacetime [11]. In the latter case the constant $B$ is negative.

If the Cardassian models are to be regarded as defining a theory of gravity then there should exist a definition of these models applying to solutions without symmetry which reduces to the known definition in the homogeneous and isotropic case. A general theory of this type is also of practical importance for comparisons with cosmological observations. Some of these comparisons use linearization around the homogeneous and isotropic models. In order to obtain the field equations for linearized perturbations the natural procedure is to start with a general theory and linearize. To the authors' knowledge no such general theory has been found. The comparison of theory with some aspects of observations, e.g. estimates of the age of the universe or supernova data, only requires the scale factor but other data such as that on cosmic microwave background fluctuations require more structure (including an 
understanding of linear perturbations) on the theoretical side to allow a comparison. The fact that calculations could be done for linearized perturbations is based on the use of what will be called the 'effective fluid' in what follows.

Because of the high symmetry of homogeneous and isotropic geometries any Cardassian model with a specific choice of $f$ and matter modelled by a perfect fluid with a specific choice of equation of state $p=g(\rho)$ is equivalent to general relativity with an effective fluid with equation of state $p=\tilde{g}(\rho)$, where $\tilde{g}$ is determined uniquely by $f$ and $g$. Starting from this observation it is possible to use the linearized Einstein-Euler equations with this effective equation of state to do perturbation theory. If, on the other hand, another specific description of matter, such as kinetic theory, is used then things are more complicated. For a particular solution of the Einstein equations coupled to this matter model which is homogeneous and isotropic it is possible to define energy density and pressure and, with a suitable monotonicity assumption, an equation of state relating the two. However this 'equation of state' depends on the solution (and hence on the initial data) and as a consequence not only on the choice of matter model. Thus it cannot be used to define a generalization to the inhomogeneous case. The unfortunate lack of a general theory cannot simply be overcome by an appeal to braneworld models or loop quantum cosmology since these constructions have also only been carried out fully under symmetry assumptions.

It will be shown that it is possible to define a natural generalization of the Cardassian models to spacetimes which are homogeneous but not necessarily isotropic. This accommodates any form of matter. For general matter models the energy-momentum tensor is intrinsically anisotropic_-it has distinct principal pressures - and so this cannot be replaced by any effective perfect fluid description. A limitation of this definition is that, as will be shown in section 3 , for the most general classes of homogeneous geometries and matter it requires the assumption that the cosmological expansion never vanishes in order to have a regular evolution equation. An interesting dynamical feature of Cardassian models in contrast to general relativity with conventional fluid is the occurrence of big rip singularities [2]. This means that the scale factor $a(t)$ tends to infinity in a finite proper time. The main result of this paper is that under general assumptions homogeneous spacetimes isotropize in the approach to a big rip singularity. This is analogous to the cosmic no hair theorem for the late-time behaviour of solutions of the Einstein equations with positive cosmological constant and its proof in the homogeneous case due to Wald [13].

The structure of the paper is as follows. Section 2 contains an analysis of the dynamics of homogeneous and isotropic Cardassian models using an effective potential. The method of an effective fluid is also described, together with its limitations. In section 3 , the generalization to cases which are homogeneous but not isotropic is presented and isotropization near a big rip singularity is proved. In the last section possible further developments of Cardassian models and related topics are discussed. This paper is based in part on the diploma thesis of the first author [1].

\section{Isotropic models}

The basic equation for Cardassian models is (5). The analysis of this section is restricted to isotropic models but spatial curvature is included so that (5) becomes

$$
\left(\frac{\dot{a}}{a}\right)^{2}=\frac{8 \pi}{3}(\rho+f(\rho))-\frac{k}{a^{2}} .
$$

The curvature parameter $k$ is $+1,0$ or -1 . Equation (4) expressing energy-momentum conservation remains unchanged. Differentiating (6) with respect to time and using (4) in 
the result gives the following evolution equation:

$$
\frac{\ddot{a}}{a}=-\frac{4 \pi}{3}(\rho+3 p)+\frac{8 \pi}{3}\left[f(\rho)-\frac{3}{2}(\rho+p) f^{\prime}(\rho)\right] .
$$

It is clear from this expression that accelerated expansion is possible even when the matter satisfies the strong energy condition. These equations for describing gravity can in principle be combined with any model of matter. The equations of motion of the matter are assumed to take exactly the same form as in general relativity. The remainder of this section will concentrate on the case where the matter is described by a perfect fluid with equation of state $p=g(\rho)$. Other matter models will be included in the discussion of anisotropic geometries in the next section.

The dynamics of isotropic models with a fluid will now be analysed. A convenient way of doing this is to use the analogy of the motion of a particle in a potential. It is assumed that the function $g$ defining the equation of state is non-negative with $g(0)=0$ and satisfies $0 \leqslant g^{\prime}(\rho) \leqslant 1$ for all values of $\rho$. Define a quantity $r$ representing the mass density of the fluid by

$$
r(\rho)=\exp \left\{\int_{1}^{\rho}(\sigma+g(\sigma))^{-1} \mathrm{~d} \sigma\right\}
$$

Under the given assumptions $0<\mathrm{d} r / \mathrm{d} \rho \leqslant 1$. From (8) it can be concluded that $r \leqslant \rho \leqslant r^{2}$ for $\rho \geqslant 1$ and $r^{2} \leqslant \rho \leqslant r$ for $\rho \leqslant 1$. It follows that the mapping from $\rho$ to $r$ can be inverted. Integrating equation (4) shows that $a^{3} r$ is time independent. Combining this with the conservation law results in a relation $\rho=h(a)$ for a function $h$ depending on $g$. In the particular case that $g$ is a linear function of $\rho$ the function $h$ is equal to a constant times a power of $a$. Define a potential $V$ by $V(a)=-\frac{4 \pi}{3} a^{2}(h(a)+f(h(a)))$. Then the dynamics of $a$ is equivalent to that of a particle in this potential. The particle has energy $-k / 2$ as a consequence of (6). The picture with the potential is useful for giving an intuitive method for seeing how the dynamics looks. It can however do more and give rigorous proofs of the main features of the dynamics. For the convenience of the reader this will be shown in detail in an appendix.

Consider the case, which may be called the standard case, where the Cardassian term is defined by $f(\rho)=B \rho^{n}$ and the equation of state of the perfect fluid is $g(\rho)=(\gamma-1) \rho$ with $1 \leqslant \gamma \leqslant 2$. Then $\rho=A a^{-3 \gamma}$ for a positive constant $A$ and

$$
V(a)=-\frac{4 \pi}{3}\left(A a^{-3 \gamma+2}+B A^{n} a^{-3 n \gamma+2}\right) .
$$

The cases $n=0$ and $n=1$ will be excluded from consideration since the first can be identified with general relativity with cosmological constant and the second is general relativity with vanishing cosmological constant in disguise. These cases are already sufficiently well known. The domain of definition of the potential is $(0, \infty)$ since $A$ is positive and $-3 \gamma+2$ is negative. The derivative $V^{\prime}(a)$ has a zero $a_{*}$ if and only if either $B>0$ and $n<\frac{2}{3 \gamma}$ or $B<0$ and $n>\frac{2}{3 \gamma}$. In both cases

$$
a_{*}=\left[\frac{B A^{n-1}(2-3 n \gamma)}{3 \gamma-2}\right]^{\frac{1}{3(n-1) \gamma}} .
$$

Important information about the potential is provided by the sign of $V^{\prime \prime}$ at the critical point.

$V^{\prime \prime}\left(a_{*}\right)=-\frac{4 \pi}{3} a_{*}^{-3 \gamma} A\left[(-3 \gamma+2)(-3 \gamma+1)+B A^{n-1}(-3 n \gamma+2)(-3 n \gamma+1) a_{*}^{-3(n-1) \gamma}\right]$. 

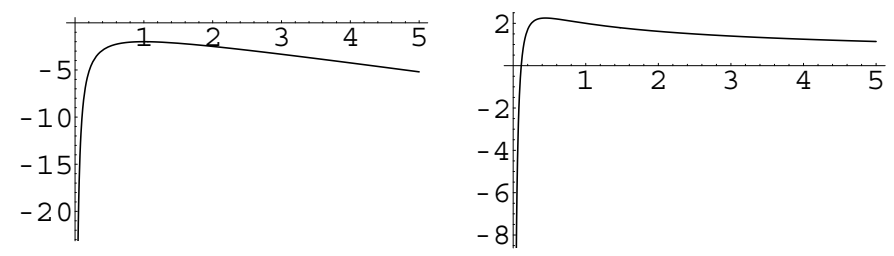

Figure 1. The cases $B>0, n<\frac{2}{3 \gamma}$ and $B<0, \frac{2}{3 \gamma}<n<1$.

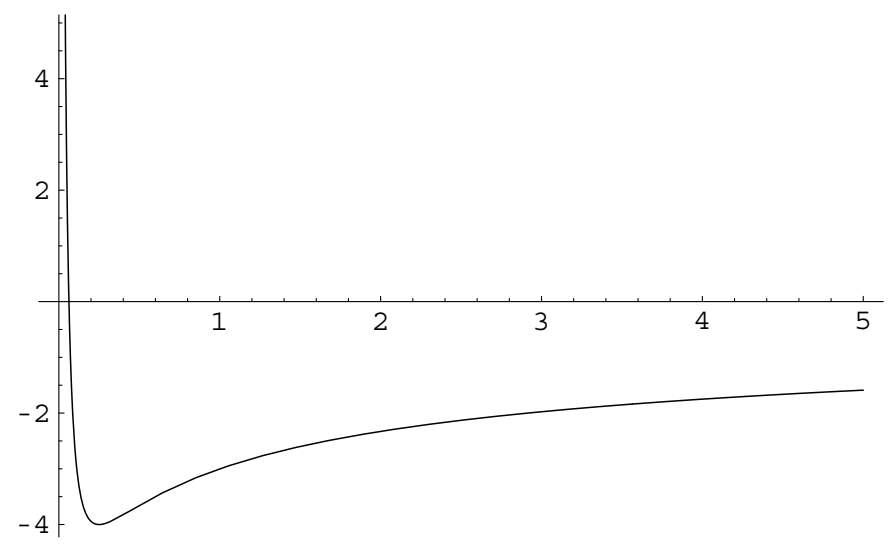

Figure 2. The case $B<0, n>1$.

From this it follows that for $n>1$ the potential has a minimum at $a_{*}$ while for $n<1$ it has a maximum there. It is now easy to read off information about the qualitative behaviour of the solution using the results of the appendix. When doing this figures 1 and 2 are useful for the purposes of orientation. In each of these figures a representative potential is shown for the given range of parameters. Since the universe is expanding today only those solutions will be considered which are expanding at some time. Note that this excludes the exceptional timeindependent solutions which occur when $B>0, n<\frac{2}{3 \gamma}$ or $B<0, n>\frac{2}{3 \gamma}$ and $k=V\left(a_{*}\right)$. The results will be summarized in a proposition.

Proposition 1. Let $a(t)$ be the scale factor of an isotropic and homogeneous solution of a Cardassian model with the matter being a perfect fluid with linear equation of state $p=(\gamma-1) \rho$ and $1 \leqslant \gamma \leqslant 2$. Suppose that there is a time $t_{0}$ with $\dot{a}\left(t_{0}\right)>0$. Then if neither $B$ nor $n-\frac{2}{3 \gamma}$ is zero the following mutually exclusive cases occur:

(1) The solution is periodic; occurs for all solutions with $B<0, n>1$ and $k>0$;

(2) a starts from zero at a finite time, increases to a maximum and returns to zero in finite time; occurs for all solutions with $B>0, n<\frac{2}{3 \gamma}, V\left(a_{*}\right)>-k / 2$ and $a<a_{*}$, all solutions with $B>0, n>\frac{2}{3 \gamma}$ and $k>0$, all solutions with $B<0, \frac{2}{3 \gamma}<n<1, V\left(a_{*}\right)>-k / 2$ and $a<a_{*}$ and all solutions with $B<0$ and $n<\frac{2}{3 \gamma}$;

(3) a tends to $\infty$ in the distant past, is decreasing before a certain time, reaches a minimum value and is increasing thereafter; occurs for all solutions with $B>0, n<\frac{2}{3 \gamma},-k / 2<$ $V\left(a_{*}\right)$ and $a>a_{*}$, all solutions with $B<0, \frac{2}{3 \gamma}<n<1,-k / 2<V\left(a_{*}\right)$ and $a>a_{*}$ and all solutions with $B<0, n>1$ and $k<0$; 
(4) a increases in a monotone way from zero at a finite time; all solutions with $B>0, n<\frac{2}{3 \gamma}$ and $-k / 2 \geqslant V\left(a_{*}\right)$, all solutions with $B>0, \frac{2}{3 \gamma}<n$ and $k<0$ and all solutions with $B<0, \frac{2}{3 \gamma}<n<1$ and $-k / 2 \geqslant V\left(a_{*}\right)$. If $-k / 2>V\left(a_{*}\right)$ then $a \rightarrow \infty$ as $t \rightarrow \infty$ while if $-k / 2=V\left(a_{*}\right)$ the solution approaches $a_{*}$ as $t \rightarrow \infty$.

The case $B=0$ corresponds to the well-known case of general relativity. If $n=\frac{2}{3 \gamma}$ the solutions behave as in the second or fourth items in the list of the proposition, depending on the value of $\frac{4 \pi A B^{n-1}}{3}$ in comparison to $-k / 2$. They are equivalent to models without Cardassian term and with a modified value of the curvature. One of the most interesting types of behaviour included in the statement of the proposition is the fact that for certain cases the big bang singularity is replaced by a bounce. It is this which, in particular, makes oscillating solutions possible.

Next, more detail will be obtained about the leading order asymptotics of the scale factor as $a$ approaches zero or infinity. Without loss of generality it may be assumed that the approach of $a$ to zero takes place in the past and its approach to infinity takes place in the future. Consider first the limit $a \rightarrow 0$. Let the degenerate case when $n=1$ and $B=-1$ be excluded from consideration. For $a$ small $V(a)=-\frac{1}{2} C^{2} a^{-2 p}(1+o(1))$ as $a \rightarrow 0$ for constants $C$ and $p$ with $p \geqslant 1 / 2$. When $a \rightarrow 0$ the curvature parameter is negligible compared to the potential and so $\dot{a}=C a^{-p}(1+o(1))$. Moreover one of the terms in the potential dominates the other. Hence

$$
a=(C(p+1))^{\frac{1}{p+1}}\left(t-t_{0}\right)^{\frac{1}{p+1}}(1+o(1)),
$$

where $t_{0}$ is the time at which $a$ vanishes. If $n>1$ the Cardassian term dominates as $a \rightarrow 0$ and putting in the relevant constants gives

$$
a=\left(12 \pi B n^{2} \gamma^{2} A^{n}\right)^{\frac{1}{3 n \gamma}}\left(t-t_{0}\right)^{\frac{2}{3 n \gamma}}(1+o(1)) .
$$

If $n<1$ the Cardassian term is negligible for $a$ small and

$$
a=\left(12 \pi \gamma^{2} A\right)^{\frac{1}{3 \gamma}}\left(t-t_{0}\right)^{\frac{2}{3 \gamma}}(1+o(1))
$$

At a big rip singularity, where $a$ tends to infinity at a finite time $t_{*}$ it is again the case that the curvature parameter becomes negligible and one of the terms in the potential dominates the other. In this case $\dot{a}=C a^{q}(1+o(1))$ with $q>0$ and $C>0$. For a finite-time singularity it is necessary that $q>1$. This corresponds to $n<0$. Letting $b=a^{-1}$ and reversing the direction of time recovers the situation analysed above, with a weaker restriction on the powers. In fact $q=1$ corresponds to $p=1$. Putting in the relevant constants allows the following asymptotics to be derived using the previous analysis:

$$
a=\left(12 \pi B n^{2} \gamma^{2} A^{n}\right)^{\frac{1}{3 n \gamma}}\left(t_{*}-t\right)^{\frac{2}{3 n \gamma}}(1+o(1)) .
$$

Here $t_{*}$ is the time at which the singularity occurs. With some more work the $o(1)$ error terms in these expressions can be replaced with specific positive powers of $t-t_{0}$ or $t_{*}-t$ respectively [1].

Now the effective fluid approach to isotropic Cardassian models will be described. The idea is to define a new energy density by $\tilde{\rho}=\rho+f(\rho)$ and a new pressure $\tilde{p}$ so that $\mathrm{d} \tilde{\rho} / \mathrm{d} t=-\frac{3 \dot{a}}{a}(\tilde{\rho}+\tilde{p})$. If this can be done and if the expression for $\tilde{\rho}$ in terms of $\rho$ can be inverted a relation of the form $\tilde{p}=\tilde{g}(\tilde{\rho})$ can be obtained. Then the dynamical properties of the Cardassian model are equivalent to that of the Einstein equations coupled to a perfect fluid with equation of state $\tilde{p}=\tilde{g}(\tilde{\rho})$. It should, however, be noted that the effective fluid has exotic properties such as violating the strong and weak energy conditions in general, the latter in the case $B<0$. In this construction the relation

$$
\tilde{p}=p+f^{\prime}(\rho)(\rho+p)-f(\rho)
$$


is obtained. To solve for $\rho$ in terms of $\tilde{\rho}$ it suffices for the condition $f^{\prime}(\rho)>-1$ to be satisfied since then the inverse function theorem can be applied. In the case of a power-law Cardassian term this works in the case $B n>0$ but not when $B n<0$. In the latter case the attempt to carry out this construction leads to an equation of state which is not single-valued. Thus this transformation cannot be carried out globally for $B n<0$. Note that in the case of forever expanding models for which $\rho \rightarrow 0$ as $t \rightarrow \infty$ the transformation can be carried out locally so as to apply to solutions at late times. When $f(\rho)=B \rho^{n}$ and $p=(\gamma-1) \rho$ the equation of state for the effective fluid approaches the linear form $\tilde{p}=(n \gamma-1) \tilde{\rho}$ as $\tilde{\rho} \rightarrow 0$. By comparison with models with a fluid with exactly linear equation of state it may be guessed that power-law inflation will occur for $n$ in the range $\left(\frac{2}{3 \gamma}, 1\right)$. This is confirmed by formula (13).

To end this section the alternative approach to Cardassian models will be considered briefly. In this case the expression $\rho+f(\rho)$ in (5) is replaced by $\rho_{1}+f\left(\rho_{1}\right)+\rho_{2}$ for two independent quantities $\rho_{1}$ and $\rho_{2}$. Here $\rho_{1}$ represents the rest energy density and $\rho_{2}$ all other forms of energy contained in the matter. The equations of motion for the matter are $\mathrm{d} \rho_{1} / \mathrm{d} t=-3 H \rho_{1}$ and $\mathrm{d} \rho_{2} / \mathrm{d} t=-3 H\left(\rho_{2}+p\right)$. In the case that $p=(\gamma-1) \rho$ the dynamics can be modelled by the potential

$$
V(a)=-\frac{4 \pi}{3}\left(A_{1} a^{-1}+B A_{1}^{n} a^{-3 n+2}\right)+A_{2} a^{-3 \gamma+2} .
$$

The critical points of this potential are not so easy to determine as those of the potential in (9) and it may not be possible to compute them explicitly. Nevertheless it should be possible to do a complete analysis of the qualitative features of the dynamics with some more work.

\section{Isotropization near big rip singularities}

There is a natural way to generalize the Cardassian models to the case of spacetimes which are homogeneous but not isotropic. The basic principle is to require the propagation of the constraint equations. The Hamiltonian constraint, which corresponds to the Friedmann equation (2) in the isotropic case, is modified when passing from general relativity to the Cardassian case in exactly the same way as was done for isotropic models - the energy density $\rho$ is replaced by $\rho+f(\rho)$ and all other terms are left unchanged. The momentum constraint is left as it is. The equations of motion of the matter, and hence the equation for energymomentum conservation, are as in general relativity. Then the question of the propagation of the constraints is posed. If both the constraints are satisfied at some time $t=t_{0}$ and a certain evolution equation generalizing (7) is satisfied at all times together with the equations of motion of the matter are the constraints satisfied at all times? It turns out that there is an evolution equation which gives a positive answer to this question and reduces to the usual evolution equation for general relativity when the Cardassian term $f$ vanishes. This equation will now be written down.

The spatially homogeneous spacetimes can be divided into the Bianchi spacetimes of types I-IX and the Kantowski-Sachs spacetimes. In the following only Bianchi models will be considered, although it would presumably be possible to carry out a similar analysis in the Kantowski-Sachs case. In the case of the Bianchi models it is possible to assume without loss of generality that the spatial manifold is simply connected. For passing from a Bianchi model to its universal cover does not change the dynamics. Once this has been done the spatial manifold can be identified with a simply connected three-dimensional Lie group $G$ and the metric and other geometrical objects can usefully be parametrized by their components in a basis of left-invariant one-forms $\theta^{i}$ on $G$ with a dual basis $e_{i}$ of vector fields. The spatial 
metric is specified by a matrix $g_{i j}(t)$ depending only on time. The spacetime metric is of the form $-\mathrm{d} t^{2}+g_{i j} \theta^{i} \theta^{j}$. Let $k_{i j}$ be the second fundamental form of the hypersurfaces of constant $t$ and define $H=-\frac{1}{3} \operatorname{tr} k$. In the isotropic case this quantity $H$ reduces to the Hubble parameter introduced previously.

The modified Hamiltonian constraint is

$$
R-k_{i j} k^{i j}+(\operatorname{tr} k)^{2}=16 \pi(\rho+f(\rho)) .
$$

The evolution equation for the second fundamental form is

$$
\frac{\mathrm{d} k_{j}^{i}}{\mathrm{~d} t}=R_{j}^{i}+(\operatorname{tr} k) k_{j}^{i}-8 \pi\left(S_{j}^{i}-\frac{1}{2} \operatorname{tr} S \delta_{j}^{i}\right)-4 \pi \rho \delta_{j}^{i}+U_{j}^{i},
$$

with trace

$$
\frac{\mathrm{d}(\operatorname{tr} k)}{\mathrm{d} t}=R+(\operatorname{tr} k)^{2}+4 \pi \operatorname{tr} S-12 \pi \rho+\operatorname{tr} U .
$$

Combining this with the generalized Hamiltonian constraint gives

$$
\frac{\mathrm{d}(\operatorname{tr} k)}{\mathrm{d} t}=\frac{1}{3}(\operatorname{tr} k)^{2}+4 \pi(\rho+\operatorname{tr} S)+\operatorname{tr} U+16 \pi f(\rho) .
$$

Here $S_{i j}=T_{i j}$ is the spatial projection of the energy-momentum tensor and

$$
U_{j}^{i}=4 \pi\left[-2 f(\rho)+f^{\prime}(\rho)\left(\rho-\frac{\nabla_{l} j^{l}}{\operatorname{tr} k}+\frac{k_{m}^{l} S^{m} l}{\operatorname{tr} k}\right)\right] \delta_{j}^{i} .
$$

In the isotropic case (21) and (22) reduce to (7). Note that $U^{i}{ }_{j}$ is in general only well defined as long as tr $k \neq 0$ since there are two terms with tr $k$ in the denominator. The first of these vanishes in Bianchi class A while the second is regular in the case of an untilted perfect fluid. (For the definitions of these terms the reader is referred to [12].) Without these restrictions it could happen that the evolution breaks down. It is however the case that for the types of solutions which will be of most interest in the following some control of the apparently singular terms can be obtained. The source of this control are some inequalities implied by the Hamiltonian constraint which will now be presented. Equation (18) can be rewritten as

$$
\frac{2}{3}(\operatorname{tr} k)^{2}=\sigma_{i j} \sigma^{i j}-R+16 \pi(\rho+f(\rho)),
$$

where $\sigma_{i j}$ is the tracefree part of $k_{i j}$. In Bianchi types I-VIII it is known that $R \leqslant 0$ [13]. Thus if a solution is of one of these Bianchi types and if $f$ is non-negative then if $\operatorname{tr} k$ tends to zero at some time it follows that $\rho$ tends to zero, with $\rho \leqslant \frac{1}{24 \pi}(\operatorname{tr} k)^{2}, f(\rho) \leqslant \frac{1}{24 \pi}(\operatorname{tr} k)^{2}$ and $\sigma_{i j} \sigma^{i j} \leqslant \frac{2}{3}(\operatorname{tr} k)^{2}$.

In spacetimes which are not isotropic the momentum constraint becomes non-trivial and must be taken into account. As has already been mentioned, for the Cardassian models it is assumed that the momentum constraint takes exactly the same form as in general relativity. This assumption is used in the calculation to verify that the Hamiltonian constraint propagates. In showing that the momentum constraint itself propagates the key feature of the modified evolution equation which is used is that the modification only affects the trace while the tracefree part of the equation remains unchanged.

It will now be investigated how solutions of these equations behave near a big rip singularity. This means by definition that the determinant det $g$ of the spatial metric or equivalently, in more geometrical language, its volume form tends to infinity in finite time. Assumptions will be made which are analogous to those made in [13]. Consider a spacetime of Bianchi type I-VIII which is expanding at some time and satisfies the Cardassian equations introduced above. Suppose further that the matter content of spacetime satisfies the dominant 
and strong energy conditions. Thus there is no dark energy included. Finally, suppose that the Cardassian term is of the form $f(\rho)=B \rho^{n}$ with $B>0$ and $n<0$.

It will now be shown that in a spacetime of this type $\operatorname{tr} k$ remains negative as long as a smooth solution exists. In fact it will be shown that as long as tr $k$ is bounded there cannot exist a sequence of times $t_{n}$ approaching the end of an interval where the solution exists for which $\operatorname{tr} k\left(t_{n}\right) \rightarrow 0$. For this purpose, suppose that there is a smooth solution defined on a time interval $\left[t_{0}, T\right)$ with $\operatorname{tr} k$ bounded and a sequence $t_{n} \rightarrow T$ with the property in question. Let $Y$ denote the right-hand side of (21). Then

$$
\operatorname{tr} k(t)=\operatorname{tr} k\left(t_{n}\right)-\int_{t}^{t_{n}} Y(s) \mathrm{d} s .
$$

Using the remarks following (23) it can be seen that all terms in $Y$ except $\operatorname{tr} U$ can be bounded by an expression of the form $C(\operatorname{tr} k)^{2}$ for a constant $C$. Under the assumption that $\operatorname{tr} k$ is bounded this implies that $\operatorname{tr} U \leqslant C|\operatorname{tr} k|$ for a constant $C$. The terms in $\operatorname{tr} U$ involving $f$ satisfy the same type of bound. It remains to consider the expressions in $Y$ where tr $k$ occurs in the denominator. In order to obtain an estimate for $\nabla_{i} j^{i}$ it is useful to evaluate it in an orthonormal frame. There the components of $j$ can be bounded by $\rho$ due to the dominant energy condition while the rotation coefficients are of order 1 . Thus $\left|\nabla_{i} j^{i}\right| \leqslant C \rho$ for a constant $C$ and $\left|\nabla_{i} j^{i}\right| /(\operatorname{tr} k) \leqslant C|\operatorname{tr} k|$. To deal with the last term in $\operatorname{tr} U$ first note that

$$
k_{m}^{l} /(\operatorname{tr} k)=\sigma_{m}^{l} /(\operatorname{tr} k)+\frac{1}{3} \delta_{m}^{l} .
$$

Thus there is an explicit contribution $\frac{4 \pi}{3} f^{\prime}(\rho) \operatorname{tr} S$ to $\operatorname{tr} U$. This is no more difficult to estimate than the terms already discussed. For the remaining quantity to be estimated it is possible to use the inequality that $\sigma^{i}{ }_{j} S^{j}{ }_{i} \leqslant\left(S_{j}^{i} S_{i}^{j}\right)^{1 / 2}\left(\sigma^{i}{ }_{j} \sigma^{j}{ }_{i}\right)^{1 / 2}$. The dominant energy condition shows that any component of $S$ in an orthonormal frame is bounded by $\rho$. Hence the last expression can be bounded by $C \rho$. Putting all this information together and writing $\tau=t_{n}-t$ gives an inequality of the form

$$
|\operatorname{tr} k|(\tau) \leqslant|\operatorname{tr} k(0)|+C \int_{0}^{\tau}|\operatorname{tr} k(s)| \mathrm{d} s .
$$

Applying Gronwall's inequality [5] and letting $n \rightarrow \infty$ gives the conclusion that $\operatorname{tr} k=0$ on the whole interval, in contradiction to the original assumptions. This proves the desired result concerning $\operatorname{tr} k$. Note that it follows from this result that $|\operatorname{tr} k|$ has a strictly positive lower bound on the given interval.

Estimates for the spacetime will be obtained with the help of a quantity $Z$. This is modelled on the quantity $S$ introduced for similar purposes in [7] which in turn was influenced by the arguments of [13].

$$
Z=3 H^{2}-8 \pi f(\rho)=8 \pi \rho+\frac{1}{2} \sigma^{i j} \sigma_{i j}-\frac{1}{2} R .
$$

Here the equality of the second and third expressions follows from the modified Hamiltonian constraint (18). The time derivative of $Z$ is given by

$$
\frac{\mathrm{d} Z}{\mathrm{~d} t}=-2 H Z-2 H\left[\sigma_{i j} \sigma^{i j}+4 \pi(\rho+\operatorname{tr} S)\right] \leqslant-2 H Z .
$$

Since it has been proved that $H>0$ it can in particular be concluded that $Z$ is non-increasing. Now introduce a quantity $l(t)$ which is positive and satisfies $l / l=H$. This is a replacement for $a$ in the isotropic case. At a big rip singularity $l$ tends to infinity. It follows from (28) that

$$
\frac{\mathrm{d}}{\mathrm{d} t}(\log Z) \leqslant-2 \frac{\mathrm{d}}{\mathrm{d} t}(\log l)
$$

Hence $\mathrm{d} / \mathrm{d} t\left(\log \left(Z l^{2}\right)\right) \leqslant 0$. As a consequence $Z \leqslant C_{1} l^{-2}$ for a positive constant $C_{1}$. It follows that $\rho \leqslant\left(C_{1} / 8 \pi\right) l^{-2}$. Now this can be put back into the modified Hamiltonian 
constraint to obtain a useful lower bound for $\dot{l} / l$, using the fact that in Bianchi types I-VIII the scalar curvature $R$ is non-positive. Compare the remarks following equation (23). There results an inequality of the form $\dot{l} \geqslant C_{2} l^{n+1}$, where the positive constant $C_{2}$ depends only on $C_{1}$ and $B$. This in turn means that $l^{-n}$ must tend to zero within a finite time only depending on $C_{2}$, provided the solution exists that long. Thus it has been shown under the given assumptions that if every solution continues to exist as long as $l$ remains bounded then every solution will end in a big rip singularity in finite time. The existence statement is dependent on the choice of matter model and will be addressed later.

It will now be shown that the geometry isotropizes in the approach to the big rip singularity in these models. This means by definition that $\sigma_{i j} \sigma^{i j} / H^{2}$ tends to zero as $t$ tends to the time $t_{*}$ where $l$ tends to infinity. Since $Z$ is bounded by a constant times $l^{-2}$ as $t \rightarrow t_{*}$ it follows from the definition of $Z$ that $\sigma_{i j} \sigma^{i j}=O\left(l^{-2}\right)$ in this limit. On the the other hand by the modified Hamiltonian constraint it follows that $H^{2} \geqslant \frac{8 \pi}{3} B l^{-2 n}$ and so the quotient of interest decays at least as fast as $l^{2 n-2}$ as $t \rightarrow t_{*}$. The quotients $\rho / H^{2}$ and $R / H^{2}$ satisfy similar estimates which says that the density becomes negligible compared to the critical density and the spatial scalar curvature becomes unimportant in the approach to the big rip singularity.

It is possible to refine these statements so as to obtain more detailed information about the asymptotics of the geometry in the limit $t \rightarrow t_{*}$. This is analogous to the refinement of Wald's theorem proved by Lee [6]. The first step is to determine the leading order asymptotics of tr $k$. From the modified Hamiltonian constraint and the estimates already obtained it follows that

$$
(\operatorname{tr} k)^{2}=24 \pi f(\rho)(1+o(1)) .
$$

Consider now the right-hand side of equation (20).

$$
R+(\operatorname{tr} k)^{2}+4 \pi \operatorname{tr} S-12 \pi \rho=(\operatorname{tr} k)^{2}(1+o(1)) .
$$

For a power law Cardassian term $\rho f^{\prime}(\rho)=n f(\rho)$. In the case of a perfect fluid with equation of state $p=(\gamma-1) \rho$ the explicit contribution containing $\operatorname{tr} S$ coming from (25) adds to $4 \pi \rho f^{\prime}(\rho)$ to give $4 \pi \gamma \rho f^{\prime}(\rho)=4 \pi n \gamma f(\rho)$. Hence

$$
12 \pi\left(-2 f(\rho)+(\rho+\operatorname{tr} S) f^{\prime}(\rho)\right)=12 \pi(n \gamma-2) f(\rho)=\frac{1}{2}(n \gamma-2)(\operatorname{tr} k)^{2}(1+o(1)) \text {. }
$$

With the information available concerning $Z$ the estimates previously obtained for the terms containing $\nabla_{i} j^{i}$ and $k^{m}{ }_{l} S_{m}^{l}$ can be improved. Putting all these facts together shows that

$$
\frac{\mathrm{d}}{\mathrm{d} t}(\operatorname{tr} k)=\frac{n \gamma}{2}(\operatorname{tr} k)^{2}(1+o(1))
$$

It follows that $\operatorname{tr} k=\frac{2}{n \gamma}\left(t_{*}-t\right)^{-1}(1+o(1))$ and that $l=l_{0}\left(t_{*}-t\right)^{\frac{2}{3 n \gamma}}$ for a constant $l_{0}$. Thus the exact leading order asymptotics for tr $k$ and $l$ have been obtained.

With tr $k$ under control the leading order asymptotics of the metric can also be determined. The starting point is the equation $\frac{\mathrm{d}}{\mathrm{d} t}\left(g_{i j}\right)=-2 g_{i l} k_{j}{ }_{j}$. It follows from this that $\frac{\mathrm{d}}{\mathrm{d} t}\left(l^{-2} g_{i j}\right)=-2 l^{-2} g_{i l} \sigma^{l}{ }_{j}$. By using some linear algebra as in [9] it is possible to obtain an estimate of the form

$$
\left\|l^{-2} g(t)\right\| \leqslant\left\|l^{-2} g\left(t_{0}\right)\right\|+\int_{t_{0}}^{t}\|\sigma(s)\|\left\|l^{-2} g(s)\right\| \mathrm{d} s .
$$

Here $t_{0}$ is some fixed time and the norms are the matrix norms of the matrices of components of the corresponding geometric objects. The norm of $\sigma$ can be estimated in terms of $\sigma_{i j} \sigma^{i j}$ for which a decay estimate in the approach to $t=t_{*}$ has already been obtained. There result bounds for the norm of $l^{-2} g$ and for the components $l^{-2} g_{i j}$. An analogous argument can be applied to bound the components $l^{2} g^{i j}$. Thus the metric $l^{-2} g_{i j}$ is uniformly equivalent to the flat metric with components $\delta_{i j}$. Using the evolution equation for $l^{-2} g_{i j}$ again shows 
that this quantity converges to a limit $g_{i j}^{*}$ as $t \rightarrow t_{*}$. Its inverse also converges so that $g_{i j}^{*}$ is non-degenerate. Finally the leading term in the expansion of the metric can be derived. The results which have been proved assuming a continuation criterion will now be summed up in a theorem.

Theorem. Consider a spatially homogeneous solution of Bianchi type I-VIII of the Cardassian equations (18) and (19) in the case that $f(\rho)=B \rho^{n}$ with $B>0$ and $n<0$. Suppose that the matter is described by a perfect fluid with equation of state $p=(\gamma-1) \rho, 1 \leqslant \gamma \leqslant 2$. If the solution is expanding at some time then the determinant of the metric tends to infinity at a finite time $t_{*}$ in the future. For $t \rightarrow t_{*}$,

$$
g_{i j}(t)=\left(t_{*}-t\right)^{\frac{4}{3 n \gamma}} g_{i j}^{*}+o\left(\left(t_{*}-t\right)^{\frac{4}{3 n \gamma}}\right)
$$

and $\operatorname{tr} k \rightarrow \frac{2}{n \gamma}$.

Note how similar this result is to that in the homogeneous case. As in that case more detailed asymptotics can be obtained if desired [1]. To complete the proof of this theorem a continuation criterion must be proved. It is contained in the following lemma.

Lemma. Under the assumptions of the theorem if the solution exists on a time interval $\left[t_{0}, t_{1}\right)$ for some finite $t_{1}$ and the determinant of the metric remains bounded as $t \rightarrow t_{1}$ then the solution can be extended to an interval $\left[t_{0}, t_{2}\right)$ for some $t_{2}>t_{1}$.

Proof. A key fact in proving this lemma is the boundedness of $Z$. The energy density satisfies the evolution equation

$$
\frac{\mathrm{d} \rho}{\mathrm{d} t}=(\operatorname{tr} k)(\rho+p)-\nabla_{i} j^{i}+\sigma^{i}{ }_{j} k_{i}^{j}=\operatorname{tr} k \gamma \rho+O(\rho) .
$$

Here it has been used that $Z$ is bounded in order to bound the term containing $\sigma^{i}{ }_{j}$. This implies that

$$
\frac{\mathrm{d}}{\mathrm{d} t}\left(\log \left(\rho l^{3 \gamma}\right)\right)=O(1)
$$

It can be concluded that since $l$ is bounded on the given interval the same is true of $\rho^{-1}$. Using the fact that $Z$ is bounded again shows that $\operatorname{tr} k$ is bounded. It is already known that $l^{-1}$ and $\rho$ are bounded. The bounds for $\operatorname{tr} k$ and $\sigma_{i j} \sigma^{i j}$ can be used to bound $g_{i j}, g^{i j}$ and $k_{i j}$ by a method which was already used above. It follows from the boundedness of tr $k$ that $(\operatorname{tr} k)^{-1}$ is also bounded, as was shown following (26). From this point the rest of the proof proceeds essentially as in section 4 of [10]. The solution remains in a compact subset of the region where the coefficients of the evolution equations for the second fundamental form are regular as a consequence of the fact that tr $k$ cannot approach zero.

The analysis of the lemma can be extended to other reasonable matter models. This includes mixtures of non-interacting fluids with linear equations of state. It also includes the case of collisionless matter satisfying the Vlasov equation. This provides a model of the distribution of galaxies which includes velocity dispersion. The only new element which comes in is the need for a suitable local existence theorem with continuation criterion. This can be proved in the same way for these matter models as in the case of a single fluid already discussed. The proof follows the arguments in section 4 of [10] as before and, in the case of collisionless matter, those of section 2 of [9]. This implies a partial analogue of the theorem for the other matter models. The statements about the occurrence of a big rip singularity and the isotropization of the geometry as it is approached go through without change. More precise information concerning the leading terms in the asymptotics depend on the details of the matter 
model chosen. For it is necessary to know the leading order behaviour of the pressure as a function of the energy density. In the case of a mixture of fluids the fluid with the smallest value of $\gamma$ will dominate. In the case of collisionless matter the leading order behaviour will be like that of dust since for collisionless matter $\operatorname{tr} S / \rho \rightarrow 0$ when the scale factor tends to infinity.

\section{Discussion}

In this paper the Cardassian models in cosmology were extended to a large class of geometries which are homogeneous but not necessarily isotropic. It was shown that for Bianchi types I-VIII, matter described by a perfect fluid with linear equation of state and Cardassian terms of the form $B \rho^{n}$ with $B>0$ and $n<0$ the property of isotropic models that all solutions develop a big rip singularity extends to all the homogeneous models. Moreover the homogeneous models approach isotropic and spatially flat models in a strong sense as the big rip singularity is approached.

At the same time it was pointed out that there is no obvious way of extending further to the inhomogeneous case so that it is not really possible to call the Cardassian models a theory of gravitation. This statement does depend on the point of view that reinterpreting the homogeneous and isotropic models in terms of an exotic fluid and passing to the inhomogeneous case in the context of the Einstein equations coupled to that fluid is not a satisfactory solution to the problem. Arguments supporting this point of view were presented. It is difficult to rule out that there is some natural general inhomogeneous version of the Cardassian models but the authors have failed to find one.

At a time when new models designed to address the question of the nature of the cause of accelerated expansion are proliferating, some of them differing from general relativity, it would be good to pose the question at an early stage of the history of such a model whether there is indeed a general theory behind it. There are cases where this is clear, as with the $f(R)$ theories which are presently very popular or other higher order theories such as those with Lagrangian $R_{\alpha \beta} R^{\alpha \beta}$. This includes the fact that the field equations for these theories are known to be well posed [8]. The example of the Cardassian models shows that things are not always so simple. It may be that the range of applicability of a proposed model does not go beyond the homogeneous case.

Big rip singularities can occur for other models which have been introduced to explain accelerated cosmological expansion. This applies in particular to general relativity coupled to certain types of exotic matter. It may well be that the isotropization phenomenon exhibited in this paper for Cardassian models also occurs in the approach to big rip singularities produced by other mechanisms. This remains to be investigated.

\section{Appendix. Motion in a potential}

Let $V$ be a smooth function defined on an interval $\left(a_{-}, a_{+}\right)$of real numbers where $a_{-}$and $a_{+}$may be finite or infinite. To exclude trivialities suppose that $V$ is not constant. Consider the equation $\ddot{a}=V^{\prime}(a)$. The energy $\frac{\dot{a}^{2}}{2}+V(a)$ is independent of time. Corresponding to initial data at some time $t_{0}$ for a solution of this equation there is a solution on a maximal interval $\left(t_{-}, t_{+}\right)$beyond which it cannot be extended smoothly. Here $t_{-}$and $t_{+}$may be finite or infinite. If one of them is finite then as $t$ tends to this endpoint $a(t)$ must come arbitrarily close to $a_{-}$or $a_{+}$. For if this were not the case, the conservation of energy would imply that $\dot{a}$ remains bounded. Then $a$ and $\dot{a}$ remain in a compact subset of the admissible values where 
the coefficients of the equation are regular and by a standard result on ordinary differential equations [5] the solution can be extended, contradicting the original assumption.

Let $a(t)$ be a solution with initial data given by $a\left(t_{0}\right)=a_{0}$ and $\dot{a}\left(t_{0}\right)=a_{1}$. Its energy is $E=\frac{1}{2} a_{1}^{2}+V\left(a_{0}\right)$. Let $b_{-}=\inf \left\{a: V(a)<E\right.$ on $\left.\left(b_{-}, a_{0}\right)\right\}$ if the set over which the infimum is to be taken is non-empty and $b_{-}=a_{0}$ otherwise. Correspondingly, let $b_{+}=\sup \left\{a: V(a)<E\right.$ on $\left.\left(a_{0}, b_{+}\right)\right\}$if the set over which the supremum is to be taken is non-empty and $b_{+}=a_{0}$ otherwise. If $b_{-}=b_{+}$then $V^{\prime}\left(a_{0}\right)=0$ and the solution is time independent. Otherwise the solution defines an interval $\left[b_{-}, b_{+}\right]$of positive length. Suppose that $b_{-}>a_{-}$and that the solution reaches the point $b_{-}$at a finite time $t_{1}>t_{0}$. Then $\dot{a}\left(t_{1}\right)=0$ and $V^{\prime}\left(b_{-}\right) \leqslant 0$. If $V^{\prime}\left(b_{-}\right)$were zero the solution could only be the time independent solution at $b_{-}$. Thus $V^{\prime}\left(b_{-}\right)<0$ and $a$ is greater than $b_{-}$at times slightly after $t_{1}$. In summary, if the solution reaches a point $b_{-}$in the interval $\left(a_{-}, a_{+}\right)$after finite time it must turn around and start increasing. The same argument applies to the evolution backwards in time. An analogous statement can be obtained with $b_{-}$replaced by $b_{+}$by a similar argument. A solution behaves in a monotone way as long as it is strictly between $b_{-}$and $b_{+}$since $\dot{a}$ can never vanish there. Thus if in a given solution $\dot{a}\left(t_{0}\right)>0$ then $\dot{a}$ will increase for all $t \geqslant t_{0}$ unless $b_{+} \in\left(a_{-}, a_{+}\right)$. In the latter case $\dot{a}$ will change sign and $a$ will start to decrease. If $\dot{a}\left(t_{0}\right)<0$ then $\dot{a}(t)$ will decrease for all $t \geqslant t_{0}$ unless $b_{-} \in\left(a_{-}, a_{+}\right)$. Similar statements apply to the past time direction. If both $b_{-}$and $b_{+}$are in $\left(a_{-}, a_{+}\right)$then there are two times $t_{1}$ and $t_{2}$ at which the solution reaches $b_{-}$. At these times the initial data are identical and so the solution is periodic, oscillating between the two endpoints $b_{-}$and $b_{+}$. Thus the following cases are possible. The solution is time independent (type 1). The solution is periodic (type 2 ). The solution is such that $\dot{a}$ changes sign exactly once (type 3 ). The solution is such that the sign of $\dot{a}$ is constant (type 4). Which of these types occur is easily decided in terms of the position of $b_{-}$and $b_{+}$. In types 1 and 2 this already determines the essential features of the dynamics. In types 3 and 4 it remains to decide the following. How does the solution behave during the monotone approach to the point $a_{-}$or $a_{+}$? It can be assumed without loss of generality that $\dot{a}(t)>0$ in the time interval of interest and approaches $a_{+}$. It should be remembered that $a_{+}$may be finite or infinite and that, independently of this, $t_{+}$may be finite or infinite. For the case being considered here to occur it must be the case that $V(a)<E$ close to $a_{+}$. By conservation of energy $\dot{a}=\sqrt{2(E-2 V(a))}$. Thus what happens depends on the details of the limiting behaviour of $V$ as $a \rightarrow a_{+}$and its relation to $E$. In the examples of relevance in this paper it is always the case that $a_{-}=0$ and $a_{+}=\infty$.

\section{References}

[1] Berndt N 2008 Dynamik von kosmologischen Modellen in verallgemeinerten Relativitätstheorien Diploma Thesis Humboldt University, Berlin

[2] Caldwell R R, Kamionkowski M and Weinberg N N 2003 Phantom energy and cosmic doomsday Phys. Rev. Lett. 91071301

[3] Freese K and Lewis M 2002 Cardassian expansion: a model in which the universe is flat, matter-dominated and accelerating Phys. Lett. B 540 1-8

[4] Gondolo P and Freese K 2002 Fluid interpretation of Cardasian expansion Phys. Rev. D 68063509

[5] Hartman P 1982 Ordinary Differential Equations (Berlin: Birkhäuser)

[6] Lee H 2004 Asymptotic behaviour of the Einstein-Vlasov system with a positive cosmological constant Math. Proc. Camb. Phil. Soc. 137 495-509

[7] Moss I and Sahni V 1986 Anisotropy in the chaotic inflationary model Phys. Lett. B 178 159-62

[8] Noakes D R 1983 The initial value formulation of higher derivative gravity J. Math. Phys. 24 1846-50

[9] Rendall A D 1994 Cosmic censorship for some spatially homogeneous cosmological models Ann. Phys. 233 82-96 
[10] Rendall A D 1995 Global properties of locally spatially homogeneous cosmological models with matter Math. Proc. Camb. Phil. Soc. 118 511-25

[11] Singh P 2006 Loop cosmological dynamics and dualities with Randall-Sundrum braneworlds Phys. Rev. D 73063508

[12] Wainwright J and Ellis G F R (ed) 1997 Dynamical Systems in Cosmology (Cambridge: Cambridge University Press)

[13] Wald R M 1983 Asymptotic behaviour of homogeneous cosmological models with cosmological constant Phys. Rev. D 28 2118-20 